\title{
Leave no one behind: inclusion of alpha-1 antitrypsin deficiency patients in COVID-19 vaccine trials
}

\author{
Chengliang Yang $\mathbb{D}^{1}$, Hedi Zhao ${ }^{2}$ and Scott J. Tebbutt $\mathbb{D}^{1,3,4}$ \\ (c) The Author(s), under exclusive licence to European Society of Human Genetics 2022
}

The coronavirus disease of 2019 (COVID-19) pandemic, caused by severe acute respiratory syndrome coronavirus 2 (SARS-CoV-2) infections, continues to present an unprecedented challenge worldwide. Emerging evidence suggests that a-1 antitrypsin (A1AT), a circulating protein with protective effects on the lung and other vital organs, plays a critical role in preventing SARS-CoV-2 infection and may be a promising therapeutic option for patients with COVID-19. A1AT deficiency (AATD) is characterized by dysfunctional or insufficient levels of A1AT. Recently, we have proposed that AATD patients are a vulnerable population for COVID-19. Patients with AATD may derive limited benefit from the current COVID-19 vaccines and continue to rely on conventional medical therapy and behavioral adaptations to mitigate the risk of infection. Unfortunately, this population has not been included in the COVID-19 vaccine clinical trials and studies have yet to characterize the safety, immunogenicity, and ultimately, the efficacy of COVID-19 vaccines for AATD patients. Re-evaluation of the COVID-19 vaccine safety and immunogenicity will further promote informed decision-making for vaccination in AATD individuals and contribute to reduce morbidity and mortality from COVID-19 infection.

European Journal of Human Genetics (2022) 30:872-874; https://doi.org/10.1038/s41431-022-01047-4

\section{INTRODUCTION}

The coronavirus disease of 2019 (COVID-19) pandemic has overwhelmed healthcare institutions globally, exhausting the limited supply of medical resources and diverting resources from the diagnosis and treatment of other dangerous diseases. In the most severe circumstances, COVID-19 has resulted in acute respiratory distress syndrome (ARDS) and fatal outcomes. To curtail the spread of the virus, some countries have declared a state of national emergency and imposed stricter containment measures including mandatory self-isolation and curfews. Effective screening and identification of vulnerable populations with significant comorbidities may mitigate the consequences of COVID-19, both at the individual level and within healthcare systems. Diabetes mellitus, hypertension, chronic kidney disease, chronic obstructive pulmonary disease (COPD), and a-1 antitrypsin deficiency (AATD), have all been implicated as comorbidities in severe acute respiratory syndrome coronavirus 2 (SARS-CoV-2) infection. In particular, AATD is hypothesized to impact the efficacy of COVID-19 vaccination and the prognosis of patients suffering from COVID-19 secondary to its well-established disease pathophysiology, by means of blunted immunity, enzymatic tissue injury, inflammation, and susceptibility to infection [1, 2]. We have previously posited such rationale for encouraging individuals with AATD to adopt protective behaviors (e.g., lifestyle changes) in addition to COVID-19 vaccine uptake [2]. In this article, the emphasis is placed on synthesizing evidence and identifying gaps in research on the long-term effects of COVID-19, key treatments, and vaccination practices in individuals with AATD.

\section{SARS-COV-2 INFECTION IN INDIVIDUALS WITH AATD}

Vaccination has emerged as a key tool for mitigating the impacts of the ongoing global pandemic. Though individuals with AATD have been identified as vulnerable populations and prioritized for vaccination in public health guidelines, they have not been included in major COVID-19 vaccine clinical trials (as reported by clinicaltrials.gov). We have recently posited that AATD patients are a vulnerable population for COVID-19 and discussed potential management strategies for these individuals, which may reduce the incidence and severity of COVID19 in AATD patients and their immediate family members [1, 2]. Portuguese and Italian research groups support the hypothesis that severe AATD patients are at higher risk of SARS-CoV-2 infection $[3,4]$. Although no specific studies of vaccine efficacy have been conducted in patients with AATD, the Alpha-1 Foundation has suggested that AATD be included within the definition of COPD and be prioritized for COVID-19 vaccination, given the high risk for COVID-19 complications and prevalence. Upwards of $32 \%$ of AATD patients have been hospitalized or required critical care, nearly double the rate of hospitalization in the general population (as reported by the Alpha-1 Foundation). Furthermore, epidemiological studies have estimated $\sim 116$ million AATD carriers (those with one $\mathrm{Pi}^{*} \mathrm{~S}$ or $\mathrm{Pi}^{*} \mathrm{Z}$ allele) and 3.4 million subjects with homozygous AATD (phenotypes $\mathrm{Pi}^{*} \mathrm{SS}, \mathrm{Pi}^{*} \mathrm{SZ}$, and $\mathrm{Pi}^{*} \mathrm{ZZ}$ ) worldwide [5]. In this context, COVID-19 vaccination practices and their relation to respiratory outcomes in AATD patients deserve greater attention and scrutiny.

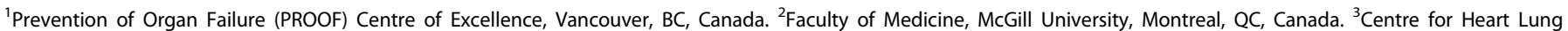

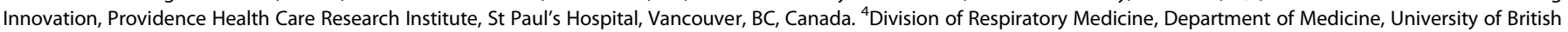
Columbia, Vancouver, BC, Canada. ${ }^{凶}$ email: Scott.Tebbutt@hli.ubc.ca
} 
AATD patients are more vulnerable to COVID-19
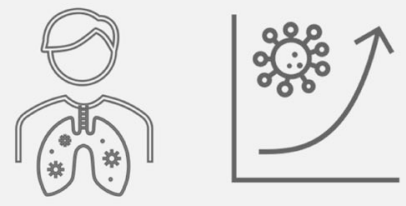

AATD patients are more likely to get long-COVID
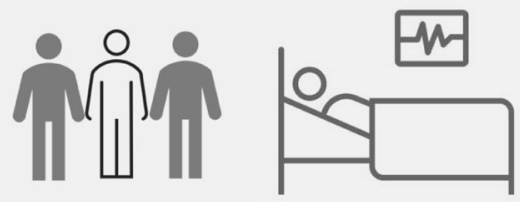

A1AT as a potential therapeutic target for COVID-19
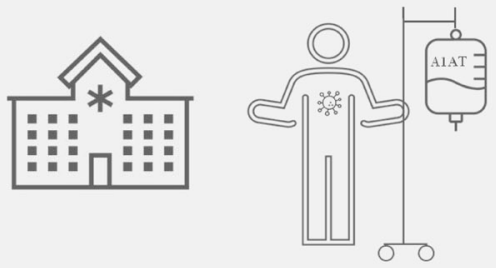

$(1)$

AATD patients should be included in clinical trials

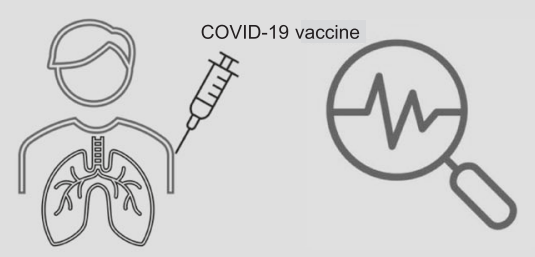

Fig. 1 Insights into COVID-19 in the context of alpha-1 antitrypsin deficiency. Proposed interactions between alpha-1 antitrypsin deficiency (AATD) therapy, long-term effects of COVID-19 (long COVID), and COVID-19 vaccines as a rationale for the inclusion of AATD individuals in future trials.

\section{AATD and long-term effects of COVID-19}

Symptoms after COVID-19 such as cough, shortness of breath, fatigue, muscle weakness, headache, palpitations, chest pain, joint pain, physical limitations, smell disorder, taste disorder, anxiety, and depression, may persist in discharged patients for at least 12 months (commonly called "the post-COVID syndrome" or as "long COVID"). Long COVID is a growing health concern. AATD is the only known inherited condition that increases the risk of developing COPD, and other chronic respiratory disorders (primarily, asthma, and bronchiectasis). Adults with pre-existing asthma or COPD present similar frequencies of depression risk (28.5\% and $32.9 \%$, respectively), COVID-19 perceived stress $(8.8 \%$ and $14.4 \%$, respectively), post-traumatic stress risk $(10.2 \%$ and $10.7 \%$, respectively), and insomnia risk $(51.8 \%$ and $60.7 \%$, respectively) [6]. Evidence has emerged that COPD may also predispose patients to long-term consequences of COVID-19, such as impaired pulmonary function, poor physical endurance, and increased post-traumatic stress [7, 8]. Asthma has similarly been associated with long COVID [9]. Notably, viral infection and COPD has a bi-directional relationship, as viral infection is a risk factor for COPD exacerbations and disease progression. As AATD is an inherited disorder caused by mutations in SERPINA1, associated with a risk of developing COPD [10] and asthma [11], it can be hypothesized that AATD individuals are at greater risk of long COVID, and this association warrants further investigation.

\section{a-1 antitrypsin as a potential therapeutic for COVID-19}

a-1 antitrypsin (A1AT) is a $52 \mathrm{kD}$ glycoprotein belonging to the serpin superfamily and is primarily synthesized in the liver and circulates in the blood. Adequate levels of A1AT are critical for the prevention of proteolytic tissue damage. The balance between proteases and antiproteases is central to lung parenchymal homeostasis, as a lack of antiproteases leads to the premature development of emphysema. Furthermore, A1AT exerts antiinflammatory and tissue-protective properties affecting a wide range of inflammatory cells. This had led to the investigation of its use as a potential therapeutic for diseases including cystic fibrosis, type 1 diabetes mellitus, graft-versus-host disease, inflammatory bowel disease, arthritis, and viral infections. Dysregulation of respiratory protease/antiprotease can also increase susceptibility to viral infection with influenza virus, human immunodeficiency virus type 1 infection, and herpes simplex virus. The pharmacokinetics and safety of A1AT have been well studied and it is considered safe, with infrequent and generally well-tolerated side effects. A1AT has shown significant promise in the treatment of COVID-19, through exerting anti-inflammatory properties and promoting cell survival in acute lung injury [12-15]. Given the aforementioned benefits, it is increasingly necessary during this time to ensure timely and adequate delivery of A1AT therapy to AATD patients.

\section{COVID-19 vaccination in the AATD population}

COVID-19 vaccines have significant potential to reduce COVID19 -associated morbidity and mortality in patients with pulmonary disease; however, for millions of individuals around the world who have AATD, it is a nuanced perspective. Since AATD contributes to pulmonary morbidity in patients with primary immunodeficiency disease, patients with AATD have an intrinsic immune system deficit which prevents them mounting a strong immune response. Because AATD patients' responses to COVID-19 vaccines may be suboptimal, continued emphasis on nonvaccine preventive measures such as lifestyle changes, pharmacotherapy, alpha-1proteinase inhibitor augmentation therapy, surgery, and other therapeutic approaches are essential, even after vaccination [2]. Moreover, vaccination of regular contacts of AATD patients may be an important adjunctive protective measure.

The main aim of this article is to highlight the absence of AATD patients in current COVID-19 vaccine trials. Until more evidence emerges, these patients remain at greater risk of contracting COVID-19 and the potentially devastating consequences. Whether AATD patients have low levels of virus-neutralizing antibody not detectable by current assays, and/or low levels of anti-SARS-CoV- 
2-specific T-cell immunity, is currently unknown, and a key question is does the impaired antibody response correspond to COVID-19 disease susceptibility? Understanding the mechanisms by which AATD patients have weakened antibody responses will complement studies on COVID-19 vaccine efficacy. Studies in solid organ transplant recipients have demonstrated insufficient immunity from the standard two-dose COVID-19 vaccine regimen, hinting of the potential for a third dose of COVID-19 as the 'best way to increase protection'. Can a third COVID-19 vaccine dose help protect AATD patients? Given the size of this potentially atrisk population, there is an urgent need to gather data to guide national and global vaccination efforts.

\section{CONCLUSION}

As the pandemic continues worldwide and resurgences in case numbers in many countries are continuing to take a toll, we believe that re-evaluation of the COVID-19 vaccine safety and immunogenicity in AATD patients will be fundamental to improving health outcomes in this high-risk population (Fig. 1). These considerations underscore the need to implement clinical trials for the development of effective vaccination strategies for individuals with AATD, including additional booster doses and different adjuvants.

\section{Take-home message}

The benefits of COVID-19 vaccination for patients with A1AT deficiency remain largely unknown and require further investigation. Re-evaluation of the COVID-19 vaccine safety and immunogenicity will further promote informed decision-making for vaccination in AATD populations.

\section{DATA AVAILABILITY}

Data sharing not applicable to this paper as no datasets were generated or analyzed during the current study.

\section{REFERENCES}

1. Yang C, Chapman KR, Wong A, Liu M. a1-Antitrypsin deficiency and the risk of COVID-19: an urgent call to action. Lancet Respir Med. 2021;9:337-9.

2. Yang C, Zhao H. COVID-19 vaccination in patients with a1-antitrypsin deficiency. Lancet Respir Med. 2021;9:818-20.

3. Faria N, Inês Costa M, Gomes J, Sucena M. Alpha-1 antitrypsin deficiency severity and the risk of COVID-19: a Portuguese cohort. Respir Med. 2021;181:106387.

4. Ferrarotti I, Ottaviani S, Balderacchi AM, Barzon V, De Silvestri A, Piloni D, et al. COVID-19 infection in severe Alpha 1-antitrypsin deficiency: Looking for a rationale. Respir Med. 2021;183:106440.

5. Greene CM, Marciniak SJ, Teckman J, Ferrarotti I, Brantly ML, Lomas DA, et al. a1Antitrypsin deficiency. Nat Rev Dis Prim. 2016;2:16051.

6. Pedrozo-Pupo JC, Campo-Arias A. Depression, perceived stress related to COVID, post-traumatic stress, and insomnia among asthma and COPD patients during the COVID-19 pandemic. Chron Respir Dis. 2020;17:1479973120962800.
7. Barh D, Tiwari S, Andrade BS, Weener ME, Góes-Neto A, Azevedo V, et al. A novel multi-omics-based highly accurate prediction of symptoms, comorbid conditions, and possible long-term complications of COVID-19. Mol Omics. 2021;17:317-37.

8. Bellan M, Soddu D, Balbo PE, Baricich A, Zeppegno P, Avanzi GC, et al. Respiratory and Psychophysical Sequelae Among Patients With COVID-19 Four Months After Hospital Discharge. JAMA Netw Open. 2021;4:e2036142.

9. Blomberg B, Mohn KG, Brokstad KA, Zhou F, Linchausen DW, Hansen BA, et al. Long COVID in a prospective cohort of home-isolated patients. Nat Med. 2021;27:1607-13.

10. Molloy K, Hersh CP, Morris VB, Carroll TP, O'Connor CA, Lasky-Su JA, et al. Clarification of the risk of chronic obstructive pulmonary disease in a1antitrypsin deficiency PiMZ heterozygotes. Am J Respir Crit Care Med. 2014;189:419-27.

11. Eden E, Mitchell D, Mehlman B, Khouli H, Nejat M, Grieco MH, et al. Atopy, asthma, and emphysema in patients with severe alpha-1-antitrypysin deficiency. Am J Respir Crit Care Med. 1997;156:68-74.

12. Yang C, Keshavjee S, Liu M. Alpha-1 Antitrypsin for COVID-19 treatment: dual role in antiviral infection and anti-inflammation. Front Pharm. 2020;11:615398.

13. Ritzmann F, Chitirala P, Krüger N, Hoffmann M, Zuo W, Lammert F, et al. Therapeutic Application of Alpha-1 Antitrypsin in COVID-19. Am J Respir Crit Care Med. 2021;204:224-7.

14. McElvaney OJ, O'Connor E, McEvoy NL, Fraughan DD, Clarke J, McElvaney OF, et al. Alpha-1 antitrypsin for cystic fibrosis complicated by severe cytokinemic COVID-19. J Cyst Fibros. 2021;20:31-5.

15. Wettstein L, Weil T, Conzelmann C, Müller JA, Groß R, Hirschenberger M, et al. Alpha- 1 antitrypsin inhibits TMPRSS2 protease activity and SARS-CoV-2 infection. Nat Commun. 2021;12:1726.

\section{AUTHOR CONTRIBUTIONS}

$\mathrm{CY}$ and SJT conceived the paper. $\mathrm{CY}$ and $\mathrm{HZ}$ conducted the search and drafted the paper. $\mathrm{CY}, \mathrm{HZ}$, and SJT revised the paper and accepted the final version for publication.

\section{FUNDING}

This study was supported by Canadian Institutes of Health Research (Grant Reference Number: 177747).

\section{COMPETING INTERESTS}

The authors declare no competing interests.

\section{ADDITIONAL INFORMATION}

Correspondence and requests for materials should be addressed to Scott J. Tebbutt.

Reprints and permission information is available at http://www.nature.com/ reprints

Publisher's note Springer Nature remains neutral with regard to jurisdictional claims in published maps and institutional affiliations. 